\title{
Image-Guided Local Therapy
}

National Cancer Institute

\section{Source}

National Cancer Institute. Image-Guided Local Therapy. NCI Thesaurus. Code C118619.

The use of imaging techniques to target treatment to a specific anatomic location or region. 